Bulletin of the Section of Logic

Volume 48/1 (2019), pp. 77-79

http://dx.doi.org/10.18778/0138-0680.48.1.05

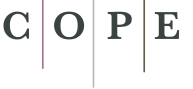

Member since 2018 JM13714

Hasan Barzegar

\title{
ERRATUM TO: CONGRUENCES AND IDEALS IN A DISTRIBUTIVE LATTICE WITH RESPECT TO A DERIVATION
}

Keywords: derivation, kernel, congruence, ideal, kernel element.

2010 Mathematics Subject Classification. 06D99, 06D15.

The present note is an Erratum for the two theorems of the paper [1]. We assume the reader is familiar with [1] and in particular with the definitions and concepts of Lattice theory.

The proof of [1, Th, 2.9] is wrong. In the end of line 10 of the proof of this theorem the equality $(x)^{d} \cap(a)^{d}=K e r d$ is not true at all. Also in line 13 the statement $a^{\prime} \in\left(x^{\prime}\right)^{d}$ iff $a^{\prime} \in\left(y^{\prime}\right)^{d}$ does not necessarily holds.

Here we have a counterexample to show this theorem is not necessarily true.

Counterexample 1. Consider the lattice $L$ as follow, $L=\left\{0, a_{1}, a_{2}, a_{3}\right.$, $\left.a_{12}, a_{13}, 1\right\}$ such that 0 and 1 are bottom and top element respectivily, $a_{1}, a_{2}$ and $a_{3}$ are attoms, $a_{1} \bigvee a_{2}=a_{12}, a_{1} \bigvee a_{3}=a_{13}, a_{2} \bigvee a_{3}=1$ and $a_{12} \bigvee a_{13}=1$. Consider the identity map $d=i d_{L}$ as a derivation on $L$. So $(a)^{d}=\{x \in L \mid a \bigwedge d(x)=0\}=\{x \in L \mid a \bigwedge x=0\}$. It is clear that $(0)^{d}=$ $L,\left(a_{1}\right)^{d}=\left\{0, a_{2}, a_{3}\right\},\left(a_{2}\right)^{d}=\left\{0, a_{1}, a_{3}, a_{13}\right\},\left(a_{3}\right)^{d}=\left\{0, a_{1}, a_{2}, a_{12}\right\}$, $\left(a_{12}\right)^{d}=\left\{0, a_{3}\right\},\left(a_{13}\right)^{d}=\left\{0, a_{2}\right\},(1)^{d}=\{0\}$ and $\mathcal{K}_{d}=\{1\}$. Thus the congruence $\theta_{d}=\left\{(x, y) \mid(x)^{d}=(y)^{d}\right\}=\Delta$ (the identity congruence). Now we introduce a congrunce $\theta$ on $L$, having $\mathcal{K}_{d}=\{1\}$ as a whole class and properly greater than $\theta_{d}$. Consider the equivalence relation $\theta$ induces by the partition $\left\{\left\{0, a_{1}\right\},\left\{a_{2}, a_{12}\right\},\left\{a_{3}, a_{13}\right\},\{1\}\right\}$. It is not difficult to check 
that the equivalence relation $\theta$ is a lattice congruence which has a $\mathcal{K}_{d}=\{1\}$ as a whole class. Clearly $\theta$ is properly greater than $\theta_{d}$.

Likewise, the Theorem 2.9 of [1] now valid only under the additional assumption with respect to the ideal $I=K e r d$. This theorem should be reformulated as:

THEOREM 2. Let $d$ be a derivation of $L$. The congruence $\theta_{d}$ is the largest congruence relation having congruence classes ker $d$ and $\mathcal{K}_{d}$, whenever $\mathcal{K}_{d} \neq \emptyset$.

Proof: First we show that $\mathcal{K}_{d}$ and ker $d$ are whole class in which the bottom element in $L / \theta_{d}$ is ker $d$ and the top element is $\mathcal{K}_{d}$ whenever $\mathcal{K}_{d} \neq \emptyset$.

Let $a \in \operatorname{ker}_{I} d$. For each $b \in \operatorname{ker}_{I} d,(a)^{d}=L=(b)^{d}$ and hence $a \theta_{d} b$. Thus $k e r_{I} d \subseteq[a]_{\theta_{d}}$. For the converse, let $c \in[a]_{\theta_{d}}$. Then $(c)^{d}=(a)^{d}=L$ and $c \in(c)^{d}$. So $d(c)=d(c \bigwedge c)=c \bigwedge d(c) \in I$ which implies $c \in k e r_{I} d$. Thus $k e r_{I} d=[a]_{\theta_{d}}$. Since $k e r_{I} d$ is an ideal of $L$, for each $[y]_{\theta_{d}} \in L / \theta_{d}$, we get that $a \bigwedge y \in k e r_{I} d$ and hence $k e r_{I} d=[a]_{\theta_{d}}=[a \bigwedge y]_{\theta_{d}} \leq[y]_{\theta_{d}}$. Therefore $k e r_{I} d$ is the bottom element in $L / \theta_{d}$. By the similar way and using the fact that if $\mathcal{K}_{d} \neq \emptyset$, then $\mathcal{K}_{d}$ is a filter, we can show $\mathcal{K}_{d}$ is the top element in $L / \theta_{d}$.

Let $\theta$ be any congruence with $\mathcal{K}_{d}$ and $K e r d$ as a congruence classes. Let $x \theta y$. Then $x \in \mathcal{K}_{d}$ iff $y \in \mathcal{K}_{d}$. If $x \in \mathcal{K}_{d}$, then $y \in \mathcal{K}_{d}$ and hence $(x)^{d}=k e r d=(y)^{d}$. Thus $x \theta_{d} y$. Now let $x \notin \mathcal{K}_{d}$ and $a \in(x)^{d}$. Then $x \bigwedge d(a)=0$ and $(x \bigwedge d(a)) \theta(y \bigwedge d(a))$. So $[y \bigwedge d(a)]_{\theta}=[0]_{\theta}=K e r d$, which implies that $d(y \bigwedge d(a))=0$. Thus $y \bigwedge d(a)=y \bigwedge d(d(a))=0$ and hence $a \in(y)^{d}$. By these conclusions we get $(x)^{d}=(y)^{d}$ and therefore $x \theta_{d} y$.

Also in line 10 of the proof of [1, Th, 3.4], the equality $d(a \bigvee b)=$ $x_{0}=x_{0} \bigvee x_{0}=d(a) \bigvee d(b)$ is wrong, indeed, $d(a)=a_{0}, d(b)=b_{0}$ and $d(a \bigvee b)=(a \bigvee b)_{0}$ which $a_{0}, b_{0}$ and $(a \bigvee b)_{0}$ not necessarily equal. The correction should be as follow.

Let $I \cap[a]_{\theta}=\left\{a_{0}\right\}$ and $I \cap[b]_{\theta}=\left\{b_{0}\right\}$. Then $(a \bigvee b) \theta\left(a_{0} \bigvee b_{0}\right)$ in which $\left(a_{0} \bigvee b_{0}\right) \in I$. So $I \cap[a \bigvee b]_{\theta}=\left\{a_{0} \bigvee b_{0}\right\}$ and hence $d(a \bigvee b)=a_{0} \bigvee b_{0}=$ $d(a) \bigvee d(b)$. 


\section{References}

[1] M. Sambasiva Rao, Congruences and ideals in a distributive lattice with respect to a derivation, Bulletin of the Section of Logic 42(1-2) (2013), pp. $1-10$.

Department of Mathematics

Tafresh University

Tafresh 3951879611, Iran

e-mail: h56bar@tafreshu.ac.ir 